Military Technical College Kobry El-Kobbah, Cairo, Egypt

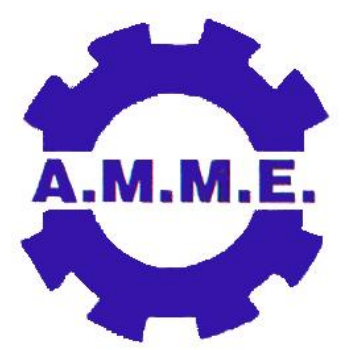

14th International Conference on Applied Mechanics and Mechanical Engineering.

\title{
ROBUST CONTROLLER'S DESIGN FOR A FUEL INJECTION PUMP
}

\author{
By \\ M. H. Alanbari $\left({ }^{*}\right) \quad$ Jaime Angulo $\left({ }^{*}\right) \quad$ José Guillermo Filippone $\left({ }^{*}\right)$ Agustín Jiménez Avello $\left({ }^{* *}\right)$
}

\begin{abstract}
:
Fuel injection pump is a practical study case of a monovariable system to get the robust controllers design in Hinf. In this application the election of the weights performance (W1(s)) and robustness sensitivity (W3(s)) depends on the hypothesis design for the robust control. Also to study the behavior of the system with the controllers designed in different values of band width with the closed loop system; at the same time, one can see the effect of uncertainty in the pattern of the system and its relationship with the robust controller's effectiveness.
\end{abstract}

\section{Keywords:}

Robust control process; Optimal control; and Application of the Hinf robust controller. 
* Department of Electro mechanics and Material of the European University in Madrid, Villaviciosa de Odón, 28670 Madrid. alanbari@.uem.es

** Department of Control and Electronic of the Polytechnic University in Madrid, José Gutiérrez Abascal, 2 - 28006 Madrid.

\section{Introduction}

Due to the continuous advance of the electronics, control systems in automotive has being made every time more complex and sophisticated. Especially there are more demands every time for an acceptable and rapid answer of control in the face of changes in the parameters of the pattern and the external atmosphere [4], [6] and [8].

This is the case of a diesel injection pump of the engine described in [3], whose diagram can be seen in figure 1 . So much servo controllers of the solenoid are immersed in the fuel, whose viscosity variation will be notably in front of the temperatures changes.

Therefore, to design a controller that has a quickly response in the face of these changes, is wanted. This problem can be solved using robust controllers design in Hinf.

\section{Model of the fuel injection pump}

The dynamic characteristics of the system have been identified to three different temperatures: $0 \mathrm{C}^{\circ}, 25 \mathrm{C}^{\circ}$ and $6 \mathrm{CC}^{\circ}$, obtaining a quite adjusted model to the physical system. Figure 2, represent the real output of the model in the case of 25Co. The transfer functions that represent the dynamic of the system in each temperature, obtained by the identification process, are the following ones. Further details can be seen in [3].

$$
\begin{aligned}
& \mathrm{G}_{0}(\mathrm{~s})=\frac{-0,01736 * s^{2}+493,9 * s^{1}-313700}{s^{3}+98,34 * s^{2}+9223 * s^{1}+87710} \\
& \mathrm{G}_{25}(\mathrm{~s})=\frac{5,498 * s^{2}+400,7 * s^{1}-444400}{s^{3}+93,72 * s^{2}+9520 * s^{1}+121400} \\
& \mathrm{G}_{60}(\mathrm{~s})=\frac{4,677 * s^{2}-285,9 * s^{1}-505300}{s^{3}+91,53 * s^{2}+10080 * s^{1}+176200}
\end{aligned}
$$




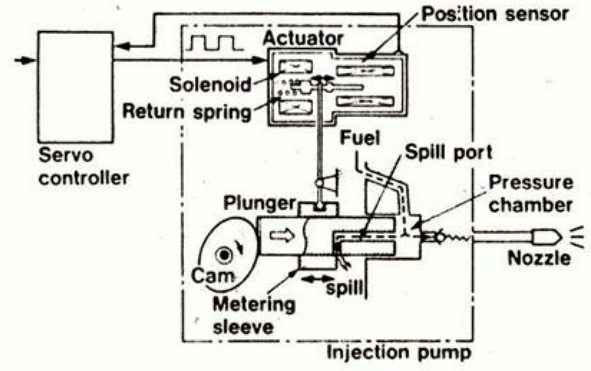

Figure (1): Fuel injection pump [3]

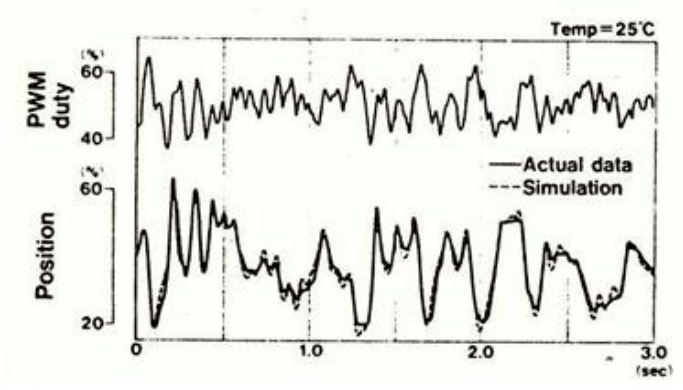

Figure (2): Real output of the system[3] 


\section{The uncertainty of the system}

The pattern $\mathrm{g}_{25}(\mathrm{~s})$ is considered as the normal pattern and the uncertainty in this pattern will be considered as a multiplicative form, that is to say:

$$
R_{m}(j w)=\Delta_{\mathrm{x}}(\mathrm{jw})=\frac{g_{x}(j w)-g_{25}(j w)}{g_{25}}
$$

for $\mathrm{x}=0 \mathrm{C}^{\circ}$ and $60 \mathrm{C}^{\circ}$

Where $R_{m}(j w)$ and $\Delta_{x}[5]$ are the uncertainty in the normal pattern.

In the figure 3 one can see the diagrams of singular values of the outputs for models family of the pump in different values of temperatures.

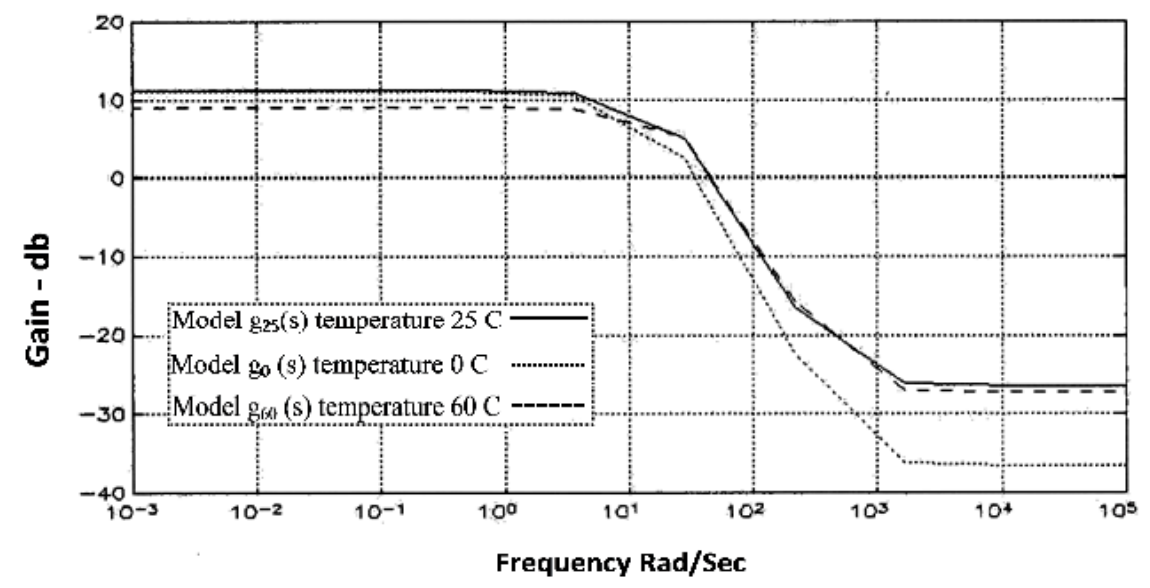

Figure (3): Transfers functions to different temperatures

\section{The objectives of the design}

The objectives of the design are to obtain a controller who has quick and efficient response to face changes in the characteristics of the servo controllers of the solenoid, due to remarkable change in the viscosity of the fuel because of the temperature changes. An application has been used to work with different bandwidth and its relationships with the degree of the robust stability for each bandwidth.

\section{Design controller F(s) in Hinf}

\section{1.- Robust stability}

The specification of robust stability is given in terms of the weight W3(s), that gives greater importance to the high frequencies and to attenuate the complementary sensitivity $T(\mathrm{~s})$ in that zone. If the normal pattern $\mathrm{G}_{25}(\mathrm{~s})$ can be stabilized in closed loop by a controller $\mathrm{F}(\mathrm{s})$, then all the models of the family $\left(\mathrm{G}_{0}(\mathrm{~s})\right.$ and $\mathrm{G}_{60}(\mathrm{~s})$ )will be able to be stabilized by the same controller if and only[2]:

$$
\|W 3(s) * T(s)\| \|_{\infty} \cong \sup |W 3(j w) * T(j w)| \leq 1 .
$$


To choose the weight W3(s) [1 and 5] so that the values of the magnitude of the uncertainty are limited to $0.3<\left|R_{m}\right|<1 / \sqrt{2}$ and the controller will be robust and works in the same rank of frequency. Is to say, that the maximum singular value of $T(s)$ is not smaller than $\sqrt{ } 2$.

Table (1): Different values of W3(s) and its Bandwidth

\begin{tabular}{|c|c|c|c|c|}
\hline Case & Values of W3 (s) & Bandwidth & Iteration number & Magnitudes of $\mathrm{R}_{\mathrm{m}}$ \\
\hline \hline 1 & $\frac{(1+S / 5)^{2}}{3,16(1+S / 500)^{2}}$ & 5 & 8 & 0.3213682 \\
\hline 2 & $\frac{(1+S / 10)^{2}}{3,16(1+S / 500)^{2}}$ & 10 & 20 & 0.3360997 \\
\hline 3 & $\frac{(1+S / 20)^{2}}{3,16(1+S / 500)^{2}}$ & 20 & 42 & 0.3949377 \\
\hline 4 & $\frac{(1+S / 30)^{2}}{3,16(1+S / 500)^{2}}$ & 30 & 55 & 0.5518112 \\
\hline 5 & $\frac{(1+S / 40)^{2}}{3,16(1+S / 500)^{2}}$ & 40 & 63 & 0.6288865 \\
\hline
\end{tabular}

The table 1 shows that the values of $R_{m}$ which are within the mentioned limits.

\section{2- The nominal performance}

The specification of the normal behaviour is represented by the weight $\mathrm{W} 1(\mathrm{~s})$, that gives greater importance to the low frequencies, so that $\mathrm{S}(\mathrm{s})$ sensitivity is small to low frequencies. The necessary and sufficient conditions to assure the normal performance of the system [2, 3 and 4]:

$$
\|W l(s) * S(s)\|_{\infty} \cong \operatorname{Sup}_{w}|W l(j w) * S(j w)| \leq 1
$$

If one wants to obtain the optimal controller that gives the best performance to the system, the following problem of optimization is created.

$$
\min _{F(s) \text { stable }}\|W l(s) * S(s)\|_{\infty}
$$

Table 2 gives different values to $\mathrm{W} 1$ (s) according to the rules published in [5], which can be applied to anyone of the cases from 1 to 5 of the table 1 , to get the best performance of the system. 
Table (2): Different values of W1(s)

\begin{tabular}{|c|c|c|c||}
\hline Case & Values of W1 (s) & Margin of gain & Margin of face \\
\hline A & $\frac{(1+S / 6)^{2}}{0,5(1+S)^{2}}$ & 3.41 & 30 \\
\hline B & $\frac{(1+S / 10)^{2}}{0,5(1+S)^{2}}$ & 5.978 & 31.3 \\
\hline C & $\frac{(1+S / 15)^{2}}{0,5(1+S)}$ & 31.41 \\
\hline D & $\frac{(1+S / 20)^{2}}{0,5(1+S)^{2}}$ & 9.54 & 28.7 \\
\hline F & $\frac{(1+S / 30)^{2}}{0,5(1+S)}$ & 7.195 & 26.7 \\
\hline G & $\frac{(1+S / 40)^{2}}{0,5(1+S)}$ & 6.069 & 26.09 \\
\hline
\end{tabular}

\section{3- The robust performance}

Once the nominal performance and the robust stability are obtained, the necessary and sufficient condition to assure the robust performance will be:

$|W 1(j w) * S(j w)|+|T(j w) * W 3(j w)| \leq 1 \quad \forall w$

or in the form of the singular values is:

$\bar{\sigma}[W l(j w) * S(j w)]+\bar{\sigma}[T(j w) * W 3(j w)] \leq 1 \quad \forall w$

So that the robust performance is guaranteed, solving the following problem in function of a scale parameter ' Gam', such that:

$$
\min _{F(s) \text { stable }} \| \operatorname{TY1v}(\mathrm{s}, \text { Gam }) \|_{\xi}=
$$

$\min _{F(s) \text { stable }}\left\|\left[\begin{array}{r}G a m * W 1(s) * S(s) \\ W 3(s) * T(s)\end{array}\right]\right\|_{\xi}$

Where TY1v (cost function), is the matrix of the transfer function of closed loop for the external signals entering to the loop and $\zeta=$ infinity

To include all what has been mentioned in the sections (2 to 5), one can put the model of fuel injection pump in the following standard form figure 4, where $P(s)$ the plants increased and the controller $\mathrm{F}(\mathrm{s})$. 


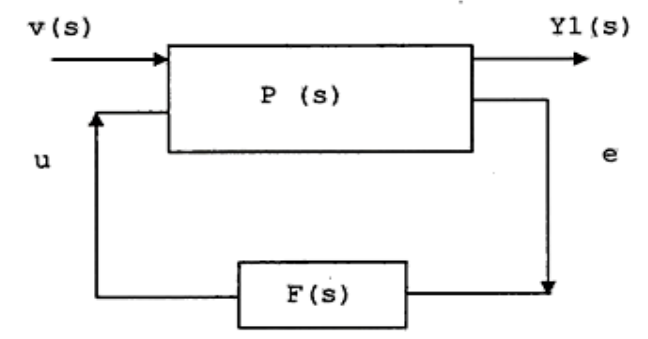

Figure (4): The system in the standard form

In order to design the robust controller in Hinf, the program designed should be applied to the nominal model g25(s) with the value of W1(s) of the table 2 case $\mathrm{C}$, and the values of W3(s) of the table 1 cases 1, 3 and 5, solving the mixed sensitivity problem of iteration on Gam until satisfies the conditions of robust performance.

\section{Results}

1- The $F(s)$ is controller with order 7 , and the Gam iteration final equal to 63.

2- Graphs of the singular values of the functions weights of the design (1/W1(s) and 1/W3(s)) in addition to the function of cost TY1v, the function sensitivity (S(s) with $1 / \mathrm{W} 1(\mathrm{~s})$ ) and the function complementary sensitivity (T(s) with $1 / \mathrm{W} 3(\mathrm{~s})$ ) for the case 5 , can be seen in figures (5 and 6), beginning with $\mathrm{Gam}=1$ and increasing as much as possible until they satisfy the conditions of robust behaviour.

3- When increasing the bandwidth, this mean increase the number of Gam-iteration necessary to assure the robust performance to obtain the robust controller $F(s)$ as it is indicated in the figure (7).

4- The figures $(9,10$ and 11) indicate the output $Y 1$ with the application of a step function. One can see the output of the system with high bandwidth (case (5)) is better compared with the (case (1)) where the bandwidth is low, motivated that the case (5) emphasizes the importance of the performance of the system in high frequency, where the magnitude of the uncertainty is greater $\left(\left|R_{m}\right|=0.6288865\right)$ contrary the case (1), which limit the knowledge of the system in high frequency and displays only the behavior of the system in law frequency where the magnitude of the uncertainty is decreased $\left(\left|R_{m}\right|=0.3213682\right)$. The weight functions $1 / \mathrm{W} 1$ y $1 / \mathrm{W} 3$ can be seen in the figure (5).

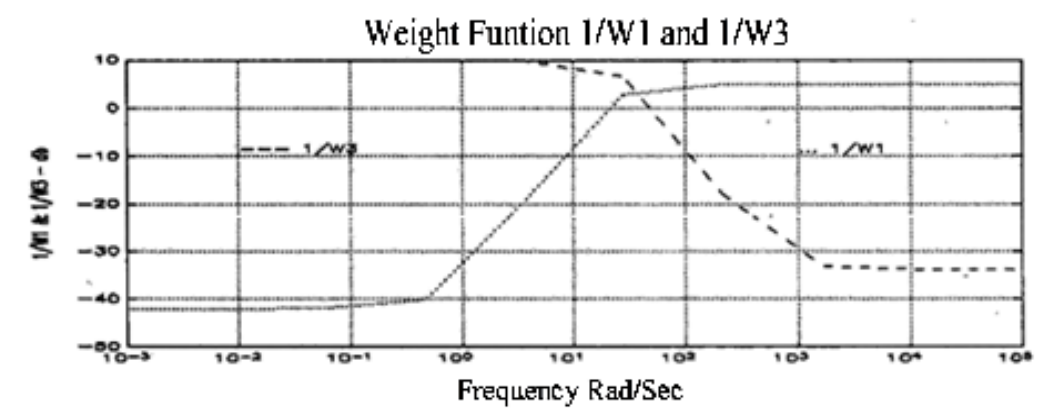

Figure (5): Singular values of the weight function of the design 
In other words that the case (5), gives the weight of the uncertainty in the model that provides more information on the behaviour of the system in high frequencies and as consequence will be greater approach to the actual plant. The behaviour of the magnitude of the uncertainty $\left|R_{m}\right|$ with the bandwidth can be seen in the figure 8 .

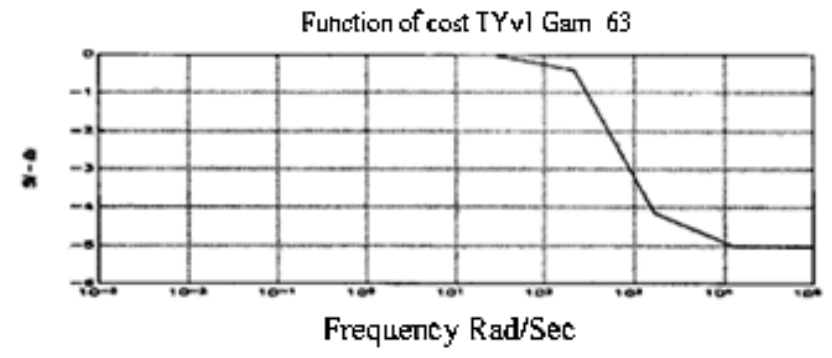

Function Sensitivity (S(s) with l/Wl(s))

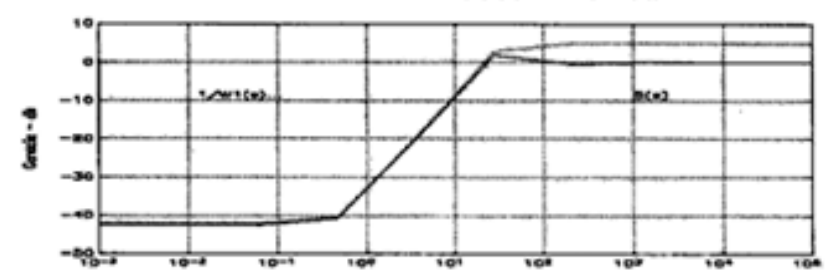

Frequency Rad/Sec

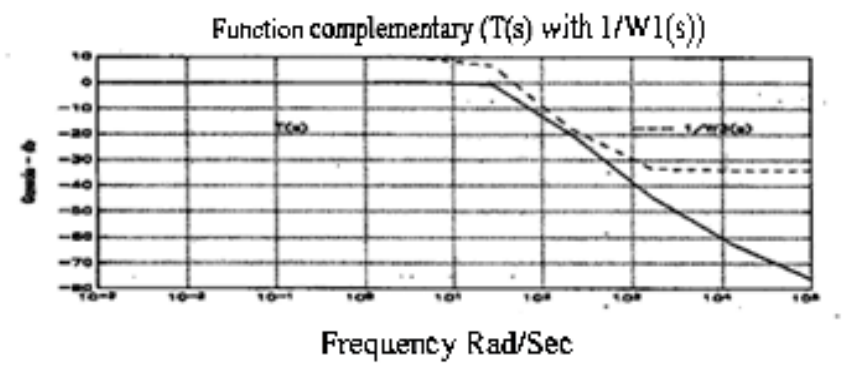

Figure (6): Function of cost TYv1, function sensitivity (S(s) with 1/W1(s)) and the function complementary sensitivity (T(s) with 1/W3(s)) for the case (5).

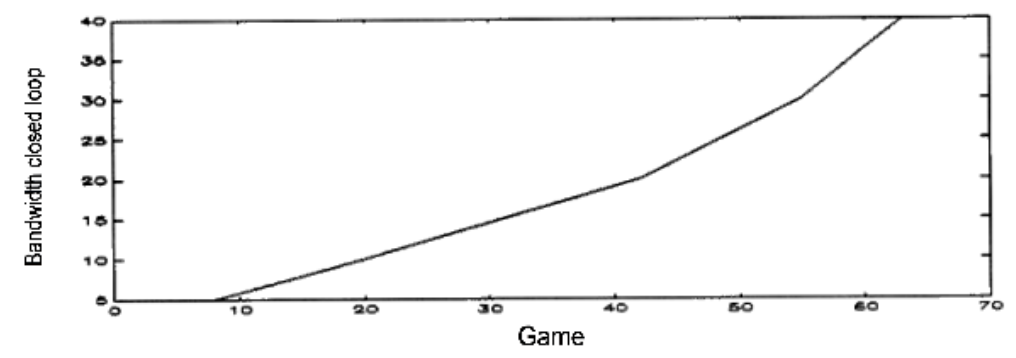

Figure (7): Bandwidth of the system in closed loop with Gam - iteration. 


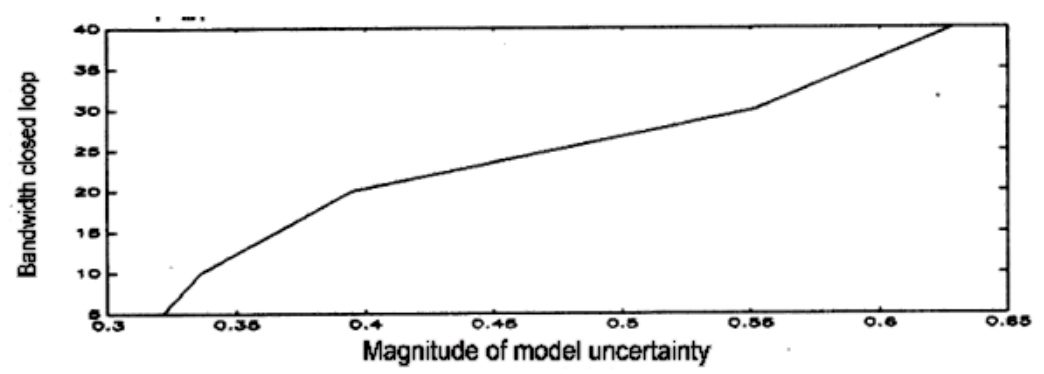

Figure (8): The bandwidth of the system in closed loop with the Rm uncertainty $R m$

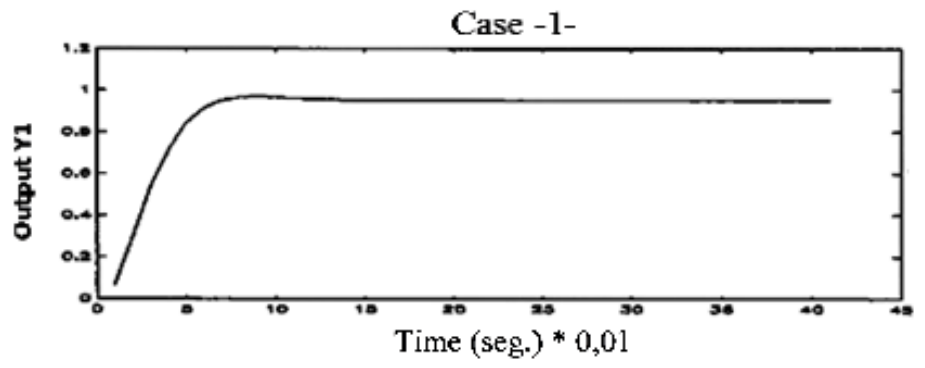

Figure (9): Output of the system Y1for the case 1

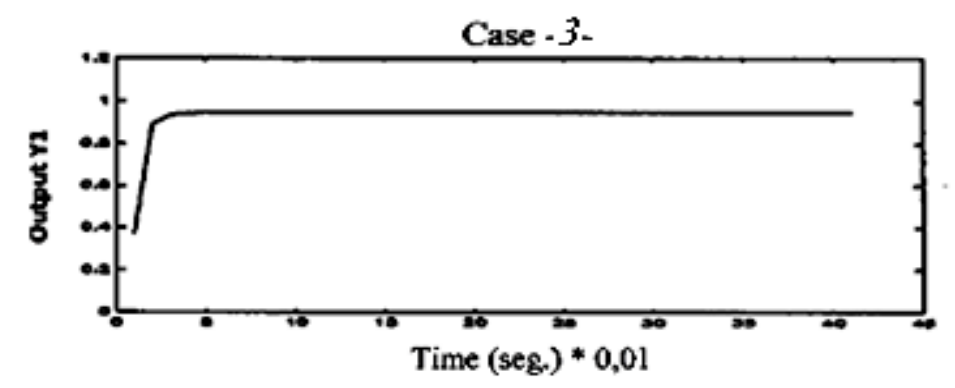

Figure (10): Output of the system Y1 for the case 3

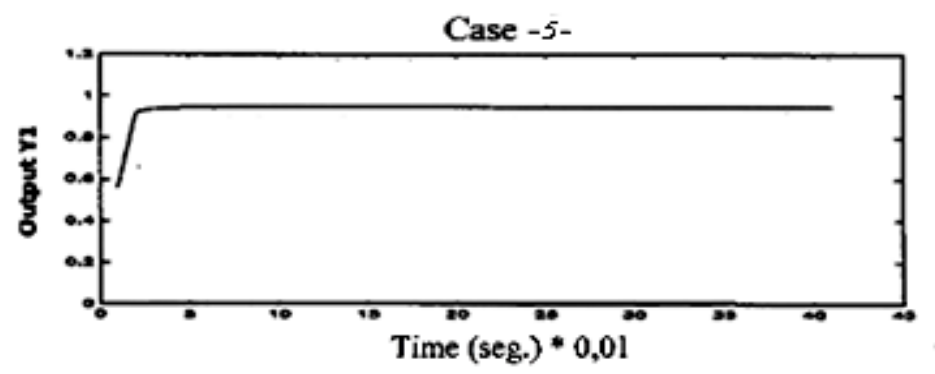

Figure (11): Output of the system Y1 for the case 5

5- In the figure (12) which indicates the robust stability and nominal behaviour of the system, with the controller designed in Hinf for the case 5. We can see that the values of the robust performance are within the limit of the necessary condition and sufficient to assure the robust behaviour of the system for these three cases, is to say $<1$. 


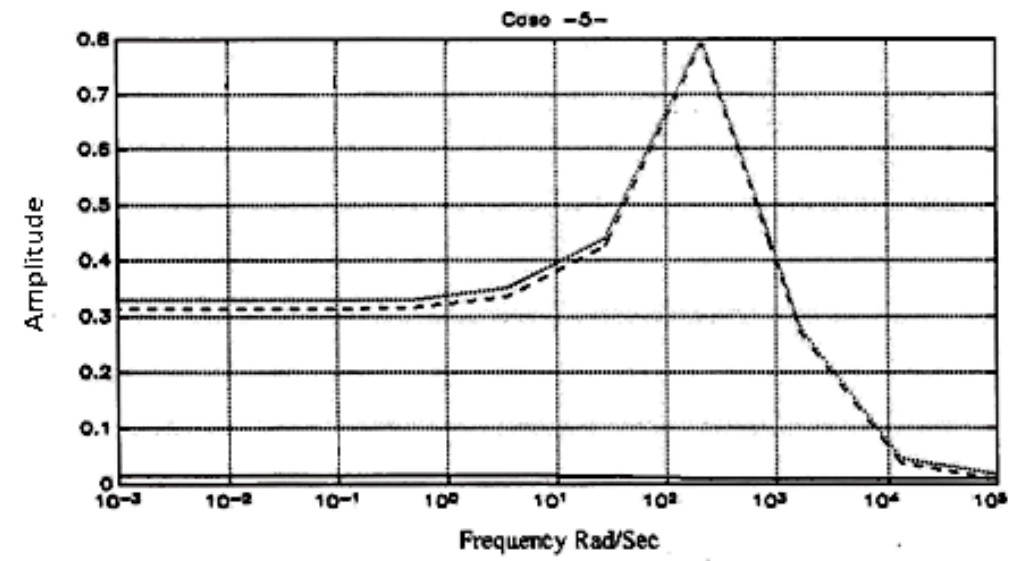

Figure (12): Conditions of the robust behavior of the controller designed in Hinf.

6- As a result of the point 5 , the $F(s)$ controller which has been applied that stabilize the nominal model $G_{25}(\mathrm{~s})$ will also stabilize all models of the family $\mathrm{G}_{0}(\mathrm{~s})$ and $\mathrm{G}_{60}(\mathrm{~s})$. The results can be seen in the figures (13 and 14) which show as the same function of sensitivity and complementary sensitivity for the nominal model $\mathrm{G}_{25}(\mathrm{~s})$ and the models of the family $\mathrm{G}_{0}(\mathrm{~s})$ and $\mathrm{G}_{60}(\mathrm{~s})$ respectively for the cases (1,3 and 5) respectively.

7-Figures (15 and 16) shows the simulated system responses in three operating temperatures using the controller designed in Hinf. It was observed that the closed-loop system is robust with respect to the changes in the temperature of the fluid, since the responses are similar which meet the design specifications.

8- Figure (17) shows the response of the system at operating temperature controller

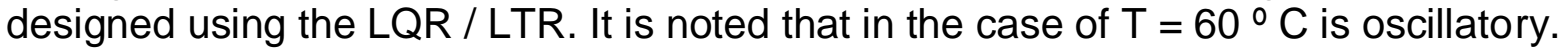


Tunctions of sensitivities case - 1 -

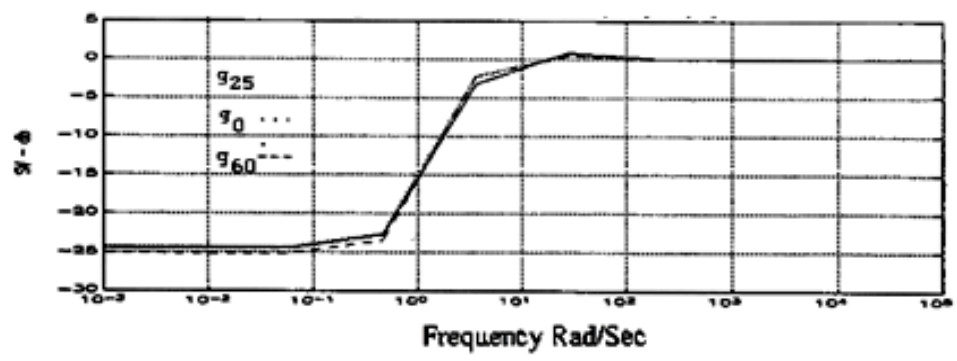

Functions of sensitivities case -3-

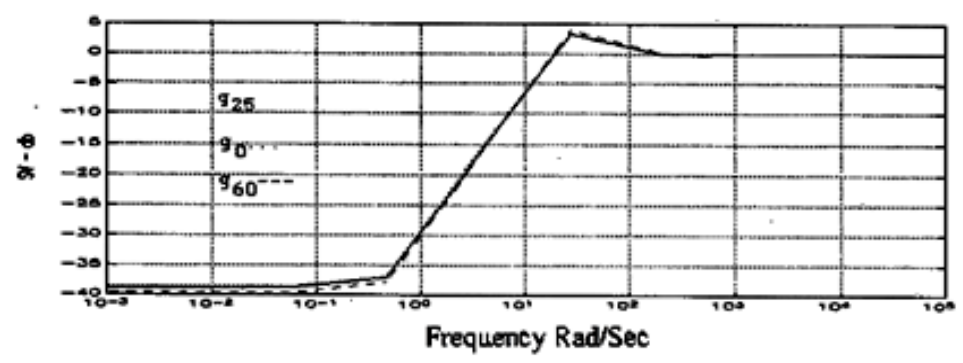

liunctions of sensitivities case -5-

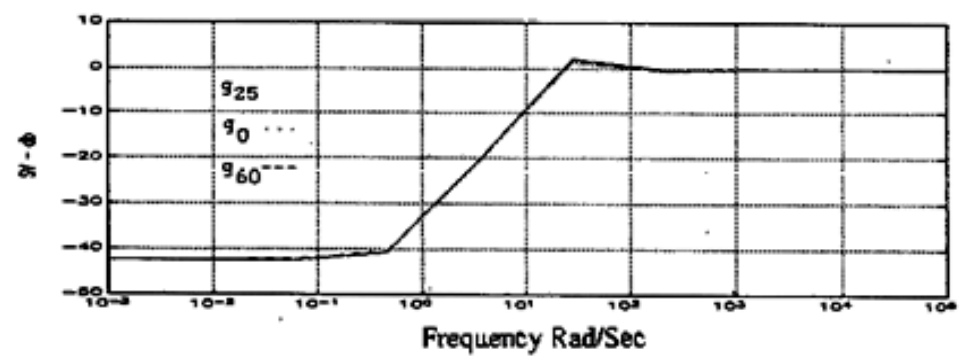

Figure (13): Functions of sensitivities of the three cases (1,3 and 5).

\section{7- Conclusions}

1-To use the controller designed by the procedure LQG / LTR the design will have to perform three different (or as many as there operating temperature) and switch the drivers in each temperature. Also should be examined experimentally in the switching transitions between the various controls, which are complicated.

2-Using the controller designed in Hinf method that optimally solves the robust stability and robust performance of a family of models with global dynamic uncertainty or nominal allowance for a family of bounded disturbances, has achieved the designed optimal controller Hinf and adequate response to three cases and presumably satisfactory intermediate temperatures $\mathrm{T} \equiv\left[0 \mathrm{C}^{\circ}, 25 \mathrm{C}^{\circ}\right.$ and $\left.60 \mathrm{C}^{\circ}\right]$.

3-Increasing the bandwidth of the system in closed loop, are minimally reduced the values of the robust behavior of the controllers designed in Hinf. 

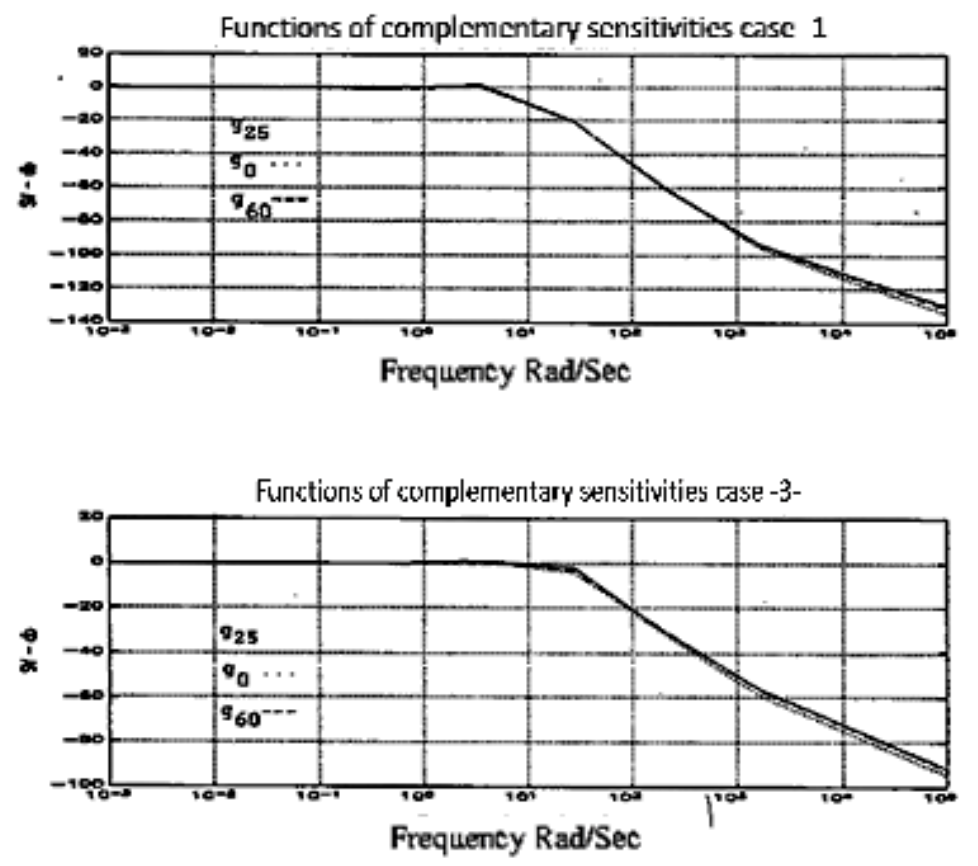

Functions of complementary sensitivities case -5-

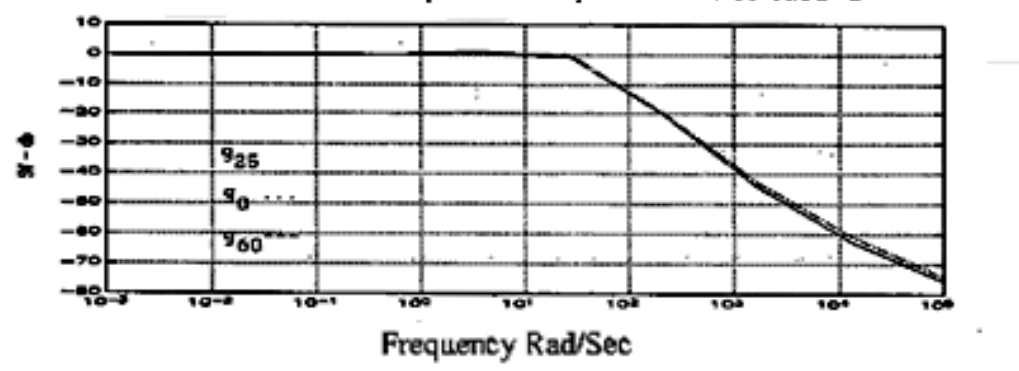

Figure (14): Functions of complementary sensitivities of the three cases (1,3 and 5)

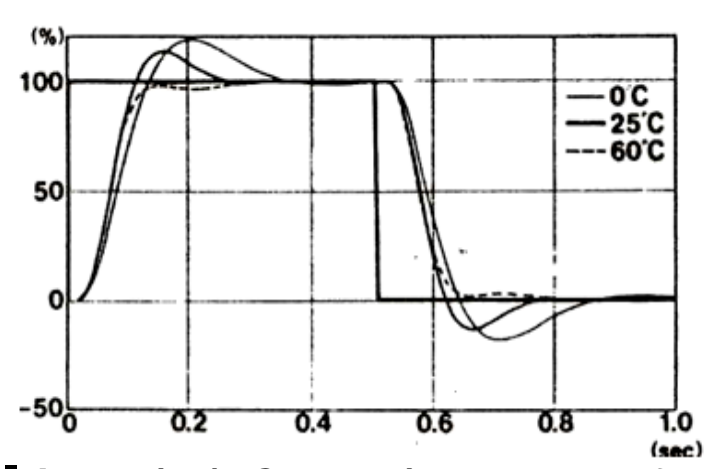

Figure (15): Control óptimo en Hinf

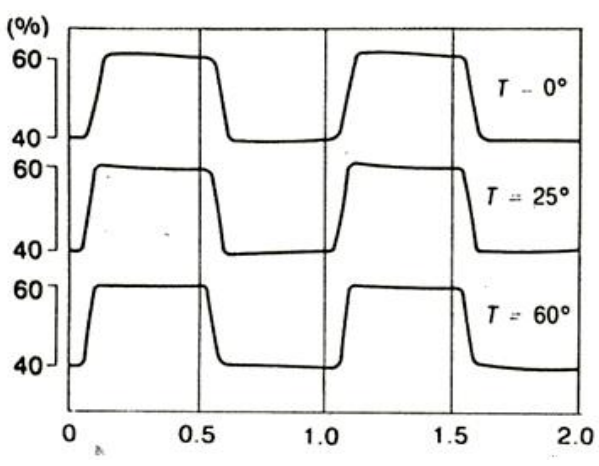

Figure (16): Hinf optimal control which can give an adequate response in all three cases 


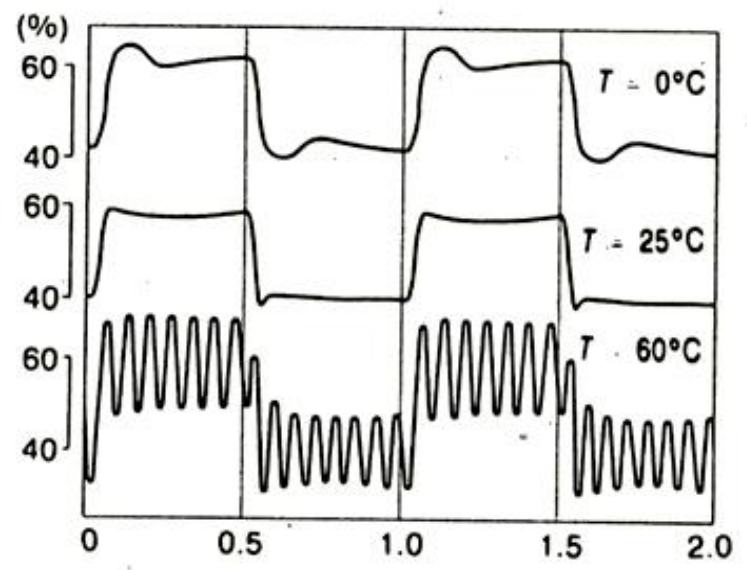

Figure (17): Control óptimo en $L Q R / L T R$ in all three cases

\section{References}

[1] C.A. Desoer, R. W. Liu, D. Murria and R. Saeks. Feedback Design: the Fractional Representation Approach to Analysis and Synthesis. IEEE Transaction on Automatic Control, 25, No.3, pp. 399-413, 1980.

[2] Glover K. and Doyle J. State Space Formula for all Stabilizing Controllers that satisfy en $H_{\infty}$ Norm Bounds and Relation to Risk Sensitivity. System and Control Letters, No. 11,1988 .

[3] Kuraoka H., Ohka N. Ohba M. Hosoe S. and Zhang F. Application of Hinfinity design to Automotive Fuel Control, IEEE Transaction on Automatic Control, 10, No.3, pp. 102-106, 1990.

[4] Larry Mianzo, Huei Peng, y Ibrahim Haskara June. Transient Aire- Fuel Ratio $\mathrm{H} \propto$ Preview Control of a Drive-By-Wire Internal Combustion Engine, Proceedings of the American Control Conference, pp. 2867 -2871,2001.

[5] M. H. Alanbari, J. G. Filippone y Agustín J. Avello Selección de Pesos de Prestación y Estabilidad para Control Robusto. XXII Jurnadas Automaticas y Control, Universidad de León, 2009.

[6] Xiao-Dong Sun and Peter G. Scotson and Graham Balfour. A Further Application of Loop Shaping Hinfinity Control to Diesel Engine Control - Driven-Idle Speed Control, Society of Automotive Engineers, Inc, 2002-01-0197.

[7] Xingquan Gao and Hong Chen, February, Constrained $\mathrm{H} \propto$ control for T-S fuzzy system and its application to inverted pendulum, IEEE Conference on Control Applications.2005.

[8] Xu S and Lam J, Robust $\mathrm{H} \propto$ control for uncertain state delayed systems with sector non-linearity, Journal of System and Control Engineering, Vol. 215, No 5, pp. 511-520, 2007. 